\title{
Unimodular Matrix with Layered Compound Structure and Its Transformation Theory
}

\author{
Hua Ma \\ College of Science, Air Force University of Engineering, Xi'an, People's Republic of China \\ Email address: \\ mahuar@xjtu.edu.cn \\ To cite this article: \\ Hua Ma. Unimodular Matrix with Layered Compound Structure and Its Transformation Theory. Mathematics Letters. \\ Vol. 6, No. 1, 2020, pp. 1-5. doi: 10.11648/j.ml.20200601.11
}

Received: May 7, 2020; Accepted: May 22, 2020; Published: May 28, 2020

\begin{abstract}
Unimodular compound structural matrix and the transformation theory are studied. Conceptually, unimodular compound structural matrix is a matrix set with layered compound structure constructed by taking some special original matrix as element and structure mode, thus having some basic properties such as unimodular, orthogonality and symmetry. Theoretically, the transformation theory of unimodular matrix have been established, using which the natural exponential matrix function of real variable and unimodular matrix can be solved efficiently; and when it is applied to the transformation of vector variable, the transformation law of variables and the invariants related to the matrix symmetry have obtained general conclusions. The results of this study are the extension of Pauli matrix, Dirac algebra and Euler equation, thus have potential applications in mathematics and physics: mathematically, which can be used as compound special matrixes to describe the compound special unitary group, to construct the algebraic structure of layered linear space, and to analytically calculate the exponential function of unimodular matrix; physically, which can be used to describe the new symmetry of intrinsic space, to express the recombination of basic particle structures, and to analysis the correlation transformation of physical mechanism.
\end{abstract}

Keywords: Unimodular Compound Matrix, Special Unitary Group, Symmetric Transformation, Intrinsic Space, Pauli Matrix, Dirac Algebra, Euler Equation

\section{Introduction}

The theory and method of matrix has become an indispensable tool in the field of modern science and technology [1, 2]. For instance, in mathematics and mathematical physics [3-6], Pauli matrix is a set of three $2 \times 2$ unitary Hermite complex matrices named physicist Wolfgang Pauli. In quantum mechanics, on the basis of Pauli matrix, Dirac matrices were introduced by Paul Dirac who was a British theoretical physicist and one of the founders of quantum mechanics. By the introduction of Dirac matrix, the problem of quantum mechanics with relativity principle was solved, and also Dirac algebra was developed. Mathematically, Dirac algebra refers to the algebraic structure in linear space constructed with 16 Dirac matrices as basis vectors, which is similar to rings in order to avoid the problem of non-conservation of probability in Klein Gordon equation. So, the problem of physics were solved, and the new physical phenomena were predicted. Obviously, under the inspiration of physical laws, physicists put forward new mathematical concepts, which not only solved physical problems, but also improved mathematical theory. In recent years, matrix theory has been further deeply and comprehensively extended to the developments of mathematics and the applications of physics [7-10].

In my researches on theoretical physics and particle physics, I have found some problems: (1) In addition to rotational symmetry, there are other symmetries in physical intrinsic space. (2) Structured description is the effective way on basic particle attributes and classification. (3) Modern science and natural phenomena were developed on the basis of basic models, which should be established from the original principles. How to solve these problems? Inspired by the works of Euler, Pauli, Dirac and other great scientists, I proposed the concept of unimodular matrix with compound structure, which can be used to describe the new symmetry of intrinsic space, to express the recombination of structural elements, and to analysis the correlation transformation of physical mechanism. 


\section{Unimodular Matrix with Compound Structure}

\subsection{Original Structural Matrix}

Firstly, taking 0,1 and -1 as the matrix elements, the original structural matrix $\tau_{i}$ is defined as follows:

$$
\begin{gathered}
\tau_{0}=\left(\begin{array}{ll}
1 & 0 \\
0 & 1
\end{array}\right), \tau_{1}=\left(\begin{array}{ll}
0 & 1 \\
1 & 0
\end{array}\right), \\
\tau_{2}=\left(\begin{array}{cc}
0 & -1 \\
1 & 0
\end{array}\right), \tau_{3}=\left(\begin{array}{cc}
1 & 0 \\
0 & -1
\end{array}\right)
\end{gathered}
$$

In the set of these matrices, $\tau_{0}, \tau_{1}, \tau_{3}$ are just the Pauli matrices, only $\tau_{2}$ uses 1 instead of the imaginary number $\mathbf{i}$ from Pauli matrix $\sigma_{2}$. Accordingly the matrix self-actions and interactions are:

$$
\tau_{i}^{2}=\left\{\begin{array}{c}
\tau_{0}, i=0,1,3 \\
-\tau_{0}, i=2
\end{array}, \tau_{1} \tau_{2}=\tau_{3}, \tau_{2} \tau_{3}=\tau_{1}, \tau_{3} \tau_{1}=-\tau_{2}\right.
$$

In order to express the above matrix relations and to facilitate the application in the compound structural matrix, an index varied label function $f(i, j)$ is induced according to the interaction relationship between matrices:

$$
\begin{aligned}
& f(1,2)=-f(2,1)=3, \quad f(2,3)=-f(3,2)=1, \quad f(3,1)=-f(1,3)=-2 \\
& f(i, i)=0 \quad(i=0,1,3), \quad f(2,2)=-0
\end{aligned}
$$

where the negative number $-i$ represent $-\tau_{i}$, namely $\tau_{-i}=-\tau_{i}$.

\subsection{Compound Structural Matrix}

Taking $\tau_{i}$ as element and structure model of compound matrix, the multi-layer model can be constructed. Using $x$ instead of element value 1 in original matrix $\tau_{i}$, derivative matrices with composite structure are:

$$
\begin{aligned}
& \tau_{0}(x)=\left(\begin{array}{ll}
x & 0 \\
0 & x
\end{array}\right), \tau_{1}(x)=\left(\begin{array}{ll}
0 & x \\
x & 0
\end{array}\right), \\
& \tau_{2}(x)=\left(\begin{array}{cc}
0 & -x \\
x & 0
\end{array}\right), \tau_{3}(x)=\left(\begin{array}{cc}
x & 0 \\
0 & -x
\end{array}\right)
\end{aligned}
$$

For instance, suppose $x=\tau_{0}, \tau_{1}, \tau_{2}, \tau_{3}$ separately, bring them in the above formula can get:

$$
\begin{aligned}
& \tau_{0}\left(\tau_{1}\right)=\left(\begin{array}{cc}
\tau_{1} & 0 \\
0 & \tau_{1}
\end{array}\right), \tau_{1}\left(\tau_{2}\right)=\left(\begin{array}{cc}
0 & \tau_{2} \\
\tau_{2} & 0
\end{array}\right), \\
& \tau_{2}\left(\tau_{3}\right)=\left(\begin{array}{cc}
0 & -\tau_{3} \\
\tau_{3} & 0
\end{array}\right), \tau_{3}\left(\tau_{0}\right)=\left(\begin{array}{cc}
\tau_{0} & 0 \\
0 & -\tau_{0}
\end{array}\right)
\end{aligned}
$$

The above definition of compound structure based matrix should be understood as double-layer structure, which can be extended to multi-layer matrix according to the same construction mechanism. In this paper, we take three layers as an example, and the compound matrix with three-layer structure is:

$$
M(i, j, k)=\tau_{i}\left(\tau_{j}\left(\tau_{k}\right)\right), i, j, k=0,1,2,3
$$

In the expression of this three-layer structure, indexes $k, j$ and $i$ identify the unit type, intermediate structure and overall framework, separately. For example:

$$
\begin{aligned}
& M(1,2,3)=\left(\begin{array}{cccc}
0 & 0 & 0 & -\tau_{3} \\
0 & 0 & \tau_{3} & 0 \\
0 & -\tau_{3} & 0 & 0 \\
\tau_{3} & 0 & 0 & 0
\end{array}\right), \\
& M(3,1,2)=\left(\begin{array}{cccc}
0 & \tau_{2} & 0 & 0 \\
\tau_{2} & 0 & 0 & 0 \\
0 & 0 & 0 & -\tau_{2} \\
0 & 0 & -\tau_{2} & 0
\end{array}\right)
\end{aligned}
$$

The corresponding specific values of these two matrix are:

$$
M(1,2,3)=\left(\begin{array}{cccccccc}
0 & 0 & 0 & 0 & 0 & 0 & -1 & 0 \\
0 & 0 & 0 & 0 & 0 & 0 & 0 & 1 \\
0 & 0 & 0 & 0 & 1 & 0 & 0 & 0 \\
0 & 0 & 0 & 0 & 0 & -1 & 0 & 0 \\
0 & 0 & -1 & 0 & 0 & 0 & 0 & 0 \\
0 & 0 & 0 & 1 & 0 & 0 & 0 & 0 \\
1 & 0 & 0 & 0 & 0 & 0 & 0 & 0 \\
0 & -1 & 0 & 0 & 0 & 0 & 0 & 0
\end{array}\right),
$$

Taking the three-layer matrix as examples, their matrix attributes and conversion properties can be derived and extended to the general matrix with more-layer structure, which include:

(1) $M(i, j, k) M(l, m, n)=M(f(i, l), f(j, m), f(k, n))$, for instance:

$$
\begin{aligned}
M(3,1,2) M(1,2,3) & =M(f(3,1), f(1,2), f(2,3)) \\
& =M(-2,3,1)=-M(2,3,1)
\end{aligned}
$$

(2) $[M(i, j, k), M(l, m, n)]_{+}=0 \quad, \quad$ on condition that $i \neq l, \quad j \neq m, \quad k \neq n$.

(3) $M(i, j, k)^{2}=M(0,0,0)=\mathbf{I}$, on condition that: $i, j, k \neq 2$ or some indexes are equal to 2 and also the number is even; $M(i, j, k)^{2}=-M(0,0,0)=-\mathbf{I}$ on condition that: some indexes are equal to 2 , and the number is odd.

(4) $\operatorname{det}(M(i, j, k))=1$. 
(5) $\operatorname{trace}(M(i, j, k))=0, \operatorname{trace}(M(0,0,0)) \neq 0$.

(6) $M(i, j, k) M(i, j, k)^{\mathrm{T}}=\mathbf{I}$, the orthogonality.

\section{Transformation Law of Unimodular Matrix}

\subsection{Expression of Exponential Function with Unimodular Matrix}

Expression theory: for an orthogonal unimodular matrix $U$, its natural exponential matrix function following with real variable $x$ can be expressed as:

$$
\mathrm{e}^{x U}=a \mathbf{I}+b U
$$

Where I is unit matrix having the same dimension with $U$, and:

$$
\left\{\begin{array}{l}
a=\frac{1}{2}\left(\mathrm{e}^{x}+\mathrm{e}^{-x}\right), \quad b=\frac{1}{2}\left(\mathrm{e}^{x}-\mathrm{e}^{-x}\right), \quad \text { when }: U^{2}=\mathbf{I} \\
a=\cos (x), \quad b=\sin (x), \quad \text { when }: U^{2}=-\mathbf{I}
\end{array}\right.
$$

Prove: According to Taylor's formula [11-13], the expression of matrix exponential function following with variable $x$ is

$$
\mathrm{e}^{x U}=\mathbf{I}+x U+\frac{(x U)^{2}}{2 !}+\frac{(x U)^{3}}{3 !}+\frac{(x U)^{4}}{4 !}+\frac{(x U)^{5}}{5 !}+\cdots
$$

When $U^{2}=\mathrm{I}$, the Taylor's expression of matrix function can be simplified to:

$$
\mathrm{e}^{x U}=\left(1+\frac{x^{2}}{2 !}+\frac{x^{4}}{4 !}+\cdots\right) \mathbf{I}+\left(x+\frac{x^{3}}{3 !}+\frac{x^{5}}{5 !}+\cdots\right) U
$$

In which, according to Taylor's formula of $\mathrm{e}^{x}$, the first term is

$$
\left(1+\frac{x^{2}}{2 !}+\frac{x^{4}}{4 !}+\cdots\right)=\frac{1}{2}\left(\left(1+\frac{x}{1 !}+\frac{x^{2}}{2 !}+\frac{x^{3}}{3 !}+\cdots\right)+\left(1+\frac{-x}{1 !}+\frac{(-x)^{2}}{2 !}+\frac{(-x)^{3}}{3 !}+\cdots\right)\right)=\frac{1}{2}\left(\mathrm{e}^{x}+\mathrm{e}^{-x}\right)
$$

The second term is

$$
\left(x+\frac{x^{3}}{3 !}+\frac{x^{5}}{5 !}+\cdots\right)=\frac{1}{2}\left(\left(1+\frac{x}{1 !}+\frac{x^{2}}{2 !}+\frac{x^{3}}{3 !}+\cdots\right)-\left(1+\frac{-x}{1 !}+\frac{(-x)^{2}}{2 !}+\frac{(-x)^{3}}{3 !}+\cdots\right)\right)=\frac{1}{2}\left(\mathrm{e}^{x}-\mathrm{e}^{-x}\right)
$$

When $U^{2}=-\mathrm{I}$, the result is just Euler formula, which is deduced by Euler according to Taylor's formula under the assumption of imaginary number $\mathrm{i}$, here $U^{2}=-\mathrm{I}$ is similar to $i^{2}=-1$.

\subsection{Invariants of Symmetric Transformation}

Conservation theory: when $U=\boldsymbol{\tau}_{i}(i=1,2,3)$, the transformation by $\mathrm{e}^{x U}$ is a symmetric transformation, and the corresponding invariants of dependent variable are determined by the symmetry of transformation matrix $\boldsymbol{\tau}_{i}$. transformation effect has its own characteristics determined by $\boldsymbol{\tau}_{i}$.

(1) For $\tau_{1}$ :

$$
A=\mathrm{e}^{x \tau_{1}}=a \mathbf{I}+b \boldsymbol{\tau}_{1}=\left(\begin{array}{ll}
a & b \\
b & a
\end{array}\right),\left(\begin{array}{l}
X^{\prime} \\
Y^{\prime}
\end{array}\right)=A\left(\begin{array}{l}
X \\
Y
\end{array}\right)=\left(\begin{array}{ll}
a & b \\
b & a
\end{array}\right)\left(\begin{array}{l}
X \\
Y
\end{array}\right)=\left(\begin{array}{l}
a X+b Y \\
b X+a Y
\end{array}\right)
$$

Thus,

$X^{\prime 2}-Y^{\prime 2}=(a X+b Y)^{2}-(b X+a Y)^{2}=\left(a^{2}-b^{2}\right)\left(X^{2}-Y^{2}\right)$, and for any real variables $x$ categorized by $\boldsymbol{\tau}_{1}$ : Suppose vector variable is $\left[\begin{array}{ll}X & Y\end{array}\right]$, matrix $A=\mathrm{e}^{x \tau_{i}}$, the

$$
a^{2}-b^{2}=\left(\frac{1}{2}\left(\mathrm{e}^{x}+\mathrm{e}^{-x}\right)\right)^{2}-\left(\frac{1}{2}\left(\mathrm{e}^{x}-\mathrm{e}^{-x}\right)\right)^{2}=\frac{1}{4}\left(\mathrm{e}^{2 x}+2 \mathrm{e}^{x} \mathrm{e}^{-x}+\mathrm{e}^{-2 x}-\mathrm{e}^{2 x}+2 \mathrm{e}^{x} \mathrm{e}^{-x}-\mathrm{e}^{-2 x}\right)=1
$$

As the result, $X^{\prime 2}-Y^{\prime 2}=X^{2}-Y^{2}$, which indicates that the invariant of transformation by $\mathrm{e}^{x \tau_{1}}$ is $X^{2}-Y^{2}$.

(2) For $\tau_{2}$ :

$$
\begin{gathered}
A=\mathrm{e}^{x \tau_{2}}=a \mathbf{I}+b \boldsymbol{\tau}_{2}=\left(\begin{array}{cc}
a & -b \\
b & a
\end{array}\right), \\
\left(\begin{array}{l}
X^{\prime} \\
Y^{\prime}
\end{array}\right)=A\left(\begin{array}{l}
X \\
Y
\end{array}\right)=\left(\begin{array}{cc}
a & -b \\
b & a
\end{array}\right)\left(\begin{array}{l}
X \\
Y
\end{array}\right)=\left(\begin{array}{l}
a X-b Y \\
b X+a Y
\end{array}\right),
\end{gathered}
$$

Thus,

$X^{\prime 2}+Y^{\prime 2}=(a X-b Y)^{2}+(b X+a Y)^{2}=\left(a^{2}+b^{2}\right)\left(X^{2}+Y^{2}\right)$, and for any real variables $x$ categorized by $\tau_{2}$ : $a^{2}+b^{2}=\cos (x)^{2}+\sin (x)^{2}=1$. So $X^{\prime 2}+Y^{\prime 2}=X^{2}+Y^{2}$, indicating that the invariant of transformation by $\mathrm{e}^{x \tau_{2}}$ is $X^{2}+Y^{2}$.

(3) For $\tau_{3}$ :

$$
\begin{gathered}
A=\mathrm{e}^{x \tau_{3}}=a \mathbf{I}+b \boldsymbol{\tau}_{3}=\left(\begin{array}{cc}
a+b & 0 \\
0 & a-b
\end{array}\right), \\
\left(\begin{array}{l}
X^{\prime} \\
Y^{\prime}
\end{array}\right)=A\left(\begin{array}{l}
X \\
Y
\end{array}\right)=\left(\begin{array}{cc}
a+b & 0 \\
0 & a-b
\end{array}\right)\left(\begin{array}{l}
X \\
Y
\end{array}\right)=\left(\begin{array}{l}
a X+b X \\
a Y-b Y
\end{array}\right) .
\end{gathered}
$$

Thus, $X^{\prime} \cdot Y^{\prime}=(a+b)(a-b) X \cdot Y=\left(a^{2}-b^{2}\right) X \cdot Y$, and for any real variables $x$ categorized by $\tau_{3}$ : 
$a^{2}-b^{2}=\left(\frac{1}{2}\left(\mathrm{e}^{x}+\mathrm{e}^{-x}\right)\right)^{2}-\left(\frac{1}{2}\left(\mathrm{e}^{x}-\mathrm{e}^{-x}\right)\right)^{2}=1$. So $\quad X^{\prime} \cdot Y^{\prime}=X \cdot Y \quad$, indicating that the invariant of transformation by $\mathrm{e}^{x \tau_{3}}$ is $X \cdot Y$.

(4) For unimodular compound structural matrix:

The transformation effects expressed by above (1), (2) and (3) also holds for the unimodular matrix $M(i, j, k)$ with composite structures. When matrix function $\mathrm{e}^{x M(i, j, k)}=a \mathbf{I}+b M(i, j, k)$ is applied to transformation on [ $\left.\begin{array}{ll}X & Y\end{array}\right]$, where $X$ and $Y$ represent multi-dimensional vector variables, and their dimensions are equal to half of the transformation matrix dimensions. Specifically, $\left[\begin{array}{ll}X & Y^{\prime}\end{array}\right]^{\mathrm{T}}=e^{x M(i, j, k)}\left[\begin{array}{ll}X & Y\end{array}\right]^{\mathrm{T}}$, and the corresponding invariants are:

$$
\text { Invariant }=\left\{\begin{array}{l}
X^{2}-Y^{2}, \quad \text { when }: i=1, \quad \text { and } j, k \neq 2 \\
X \cdot Y, \text { when }: i=3, \text { and } j, k \neq 2 \\
X^{2}+Y^{2}, \quad \text { when }: i=2, \quad \text { or one of } j, k=2
\end{array}\right.
$$

\subsection{Computational Verification}

Example 1: in a 8 dimensional intrinsic vector space, assume that $X=\left[\begin{array}{llll}1 & 2 & 3 & 4\end{array}\right]$ and $Y=\left[\begin{array}{llll}5 & 6 & 7 & 8\end{array}\right]$. When $\left[\begin{array}{ll}X & Y\end{array}\right]^{\mathrm{T}}$ is transformed by $M(1,2,3)$, what are the results? The calculations are as follows:

$$
\left[\begin{array}{ll}
X^{\prime} & Y^{\prime}
\end{array}\right]^{\mathrm{T}}=M(1,2,3)\left[\begin{array}{ll}
X & Y
\end{array}\right]^{\mathrm{T}}=\left[\begin{array}{llllllll}
-7 & 8 & 5 & -6 & -3 & 4 & 1 & -2
\end{array}\right]^{\mathrm{T}} \text {. }
$$

Indicating that: the transformation of the first level was finished by $\boldsymbol{\tau}_{1}$, results in that $X$ and $Y$ displace each other; the transformation of the second and third levels were finished by $\boldsymbol{\tau}_{2}$ and $\boldsymbol{\tau}_{3}$ respectively, results in that the displacement and inversion of the corresponding positions are transformed. The conservation: $\quad M(1,2,3)^{2}=M(0,-0,0)=-M(0,0,0)=-\mathbf{I} \quad$ ， $M(1,2,3)=\exp (x M(1,2,3))=a \mathbf{I}+b M(1,2,3)$, so $a=0, b=1$, which satisfy the condition that $a^{2}+b^{2}=\cos (x)^{2}+\sin (x)^{2}=1$. Theoretically, the invariant of transformation is $X^{2}+Y^{2}$, which are in good agreement with the calculation results: $X^{2}+Y^{2}=204, X^{\prime 2}+Y^{\prime 2}=204$.

Example 2: in a 8 dimensional intrinsic space, assume that $X=\left[\begin{array}{llll}1 & 2 & 3 & 4\end{array}\right], Y=\left[\begin{array}{llll}5 & 6 & 7 & 8\end{array}\right], x=4.8$ and $A=\exp (x M(3,1,0))$. When $\left[\begin{array}{ll}X & Y\end{array}\right]^{\mathrm{T}}$ is transformed by $A$, what are the results? The calculations are as follows:

$$
\begin{aligned}
& {\left[\begin{array}{ll}
X^{\prime} & Y^{\prime}
\end{array}\right]^{\mathrm{T}}=\exp (4.8 M(3,1,0))\left[\begin{array}{ll}
X & Y
\end{array}\right]^{\mathrm{T}}}
\end{aligned}
$$

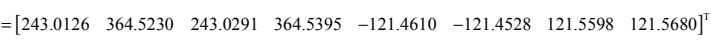

Because that: $M(3,1,0)^{2}=M(0,0,0)=\mathbf{I} \quad, \quad a=60.7593$, $b=60.7511$, and $\operatorname{det}(A)=1$. So $a^{2}-b^{2}=1$, the invariant of transformation is $X \cdot Y$. The calculated results are in good agreement with the theoretical ones: $X \cdot Y=70, X^{\prime} \cdot Y^{\prime}=70$.

\section{Summary}

The concept and transformation theory of unimodular compound structural matrix are found and put forward in the research of theoretical physics and particle physics. In terms of contents and models, this is an extension of Pauli matrix and Dirac algebra. In applications, this can be used to describe the new symmetry of intrinsic space, to express the recombination of structural elements, and to analysis the correlation transformation of physical mechanism. For the reliabilities, some correctness are proved reasonably in theory, and some correctness are verified by numerical calculations. Next, the completeness and application scope should be deeply studied.

\section{Acknowledgements}

The author is grateful to the support from the National Natural Science Foundation of China (Grant No. 61671467), and the Innovation Team of Shaanxi province (Grant No. 2014KCT-05).

\section{References}

[1] David S. Watkins, Fundamentals of Matrix Computations (Wiley, 2013).

[2] Riley, Kenneth F., Hobson, Michael P., Bence, Stephen J., Mathematical methods for physics and engineering (Cambridge University Press, 1997).

[3] J. J. Sakurai, Modern Quantum Mechanics (Massachusetts: Addison-Wesley, 1995).

[4] D. McMahon, Quantum Mechanics Demystified (Mc Graw Hill (USA), 2006).

[5] F. Mandl, G. Shaw, Quantum Field Theory (John Wiley \& Sons, 1993).

[6] Wherrett, Brian S., Group Theory for Atoms, Molecules and Solids (Prentice-Hall International, 1987).

[7] Benjamin Passer, Shape, scale, and minimality of matrix ranges. Trans. Amer. Math. Soc. 372 (2019) 1451-1484.

[8] Braxton O. and Dong W., A diffusion generated method for orthogonal matrix-valued fields. Math. Comp. 89 (2020) 515-550.

[9] Adel A., Hamed A., S. K. Jain and Efim Z.. Matrix wreath products of algebras and embedding theorems. Trans. Amer. Math. Soc. 372 (2019) 2389-2406.

[10] Mikael L., Santeri M. and Niklas W. Norm estimates of weighted composition operators pertaining to the Hilbert matrix. Proc. Amer. Math. Soc. 147 (2019) 2425-2435.

[11] Brown, William A., Matrices and vector spaces (New York, NY: M. Dekker, 1991, ISBN 978-0-8247-8419-5). 
[12] Philip N. Klein, Coding the Matrix: Linear Algebra through Applications to Computer Science (Newtonian Press, 2013).
[13] Rajendra Bhatia, Positive definite matrices (Princeton Series in Applied Mathematics, 2007). 